\title{
Emission and focusing characteristics of volcano-structured double-gated field emitter arrays
}

\author{
Yoichiro Neo, ${ }^{\text {a) }}$ Masafumi Takeda, and Takashi Soda \\ Research Institute of Electronics, Shizuoka University, 3-5-1 Johoku, Naka-ku, Hamamatsu 432-8011, Japan \\ Masayoshi Nagao, Tomoya Yoshida, and Seigo Kanemaru \\ National Institute of Advanced Industrial Science \& Technology, 1-1-1 Umezono, Tsukuba 305-8568, Japan \\ Toshikatsu Sakai, Kei Hagiwara, and Nobuo Saito \\ NHK Science \& Technical Research Laboratories, 1-10-11 Kinuta, Setagaya 157-8510, Japan \\ Toru Aoki and Hidenori Mimura \\ Research Institute of Electronics, Shizuoka University, 3-5-1 Johoku, Naka-ku, Hamamatsu 432-8011, Japan
}

(Received 3 October 2008; accepted 9 February 2009; published 27 March 2009)

\begin{abstract}
Volcano-structured double-gated field emitter arrays (VDG-FEAs) with different focusing electrode height have been fabricated and the focusing characteristics for each VDG-FEAs were evaluated in detail. The authors experimentally proved that lowering the focusing electrode was very effective to keep a high emission current and successfully removed the electric field relaxation at the tip during the focusing operation. The anode current of about $1.9 \mu \mathrm{A}$ was maintained for the VDG-FEA with the height of the focusing electrode relative to the extraction electrode, $h f=-470 \mathrm{~nm}$, even in applying $5 \mathrm{~V}$ to the focusing electrode, and the current was 84.4 times larger, compared with the sample with $h f=220 \mathrm{~nm}$ under the same focusing condition. It was found that the emitted electrons returned back to the extraction electrode by the potential barrier caused by the low focusing potential below $3 \mathrm{~V}$ in every sample. (C) 2009 American Vacuum Society.
\end{abstract}

[DOI: $10.1116 / 1.3093891]$

\section{INTRODUCTION}

The next television system, dubbed as "superhigh vision," has been proposed and developed. The superhigh vision display has $4096 \times 7680$ pixels and the pixel size should be less than $50 \mu \mathrm{m}$, assuming $50 \mathrm{in}$. in diagonal. Field emission displays (FEDs) are a promising candidate, because FEDs have many superior features, such as high picture quality, excellent motion picture, low power consumption, and wide viewing angle. However, the pixel size less than $50 \mu \mathrm{m}$ cannot be realized by conventional cone-type field emitters such as Spindt-type emitters because the conventional emitters have a relatively large beam emission angle. Therefore, several double-gated FEAs with a focusing lens structure have been proposed and beam focusing was demonstrated. ${ }^{1-3}$ However, when a lower voltage than the extraction electrode voltage is applied to the focusing electrode under focusing operation, the electric field at the tip is certainly influenced and relaxed by the low focusing potential, and as a result the emission current was significantly decreased. To control the electric field relaxation, we have fabricated the volcano-structured double-gated field emitter arrays (VDG-FEAs) and measured focusing characteristics. ${ }^{4,5}$ We have fabricated three kinds of VDG-FEAs with different height $(h f)$ of the focusing electrode relative to the focusing electrode, $h f=+200 \mathrm{~nm}, 0 \mathrm{~nm}$, and $-470 \mathrm{~nm}$. This article describes the details of the focusing characteristics and dependence of the electric field at the tip on $h f^{4}$.

\footnotetext{
a) Author to whom correspondence should be addressed; electronic mail: y-neo@rie.shizuoka.ac.jp
}

\section{EXPERIMENTS AND RESULTS}

\section{A. Structure and emission characteristics of VDG-FEAs}

Figure 1 shows the cross sectional scanning electron microscope (SEM) images of three kinds of VDG-FEAs. These VDG-FEAs were fabricated by using etch-back technique. ${ }^{6}$ The emitter tip was coated with a $15 \mathrm{~nm}$ HfC layer. The HfC coating lowers the operation voltage of the emitter and extends the operation lifetime. ${ }^{7,8}$ The height of the extraction electrode was adjusted as the same as the height of the tip. The diameter of the extraction gate hole was about $0.5 \mu \mathrm{m}$. $\mathrm{A}, \mathrm{B}$, and $\mathrm{C}$ represent the VDG-FEAs with $h f=+220 \mathrm{~nm}$, $0 \mathrm{~nm}$, and $-470 \mathrm{~nm}$, respectively. A, B, and C have 37, 19, and 7 tips, respectively, which are located in a honeycomb structure, as shown in Fig. 1. The fabrication process is shown in the previous report. ${ }^{1}$ Each leak current between the substrate, extraction electrode, and focusing electrode was less than $10 \mathrm{nA}$ and could be ignorable in the following measurements.

Each VDG-FEA was mounted on a TO-5 header. Electron emission current and focusing characteristics were measured in an ultrahigh vacuum chamber at a pressure of 1.2 $\times 10^{-7} \mathrm{~Pa}$. The anode was a phosphor $(\mathrm{ZnO}: \mathrm{Zn})$ coated screen, which was located about $2 \mathrm{~mm}$ apart from the VDGFEA. The VDG-FEA was connected to the ground through a series resistor of $100 \mathrm{k} \Omega$. The anode was constantly biased at $1 \mathrm{kV}$.

Figure 2 shows the anode currents $\left(I_{a}\right)$ for the VDG-FEAs as a function of the extraction electrode voltage $\left(V_{\text {ext }}\right)$ with- 


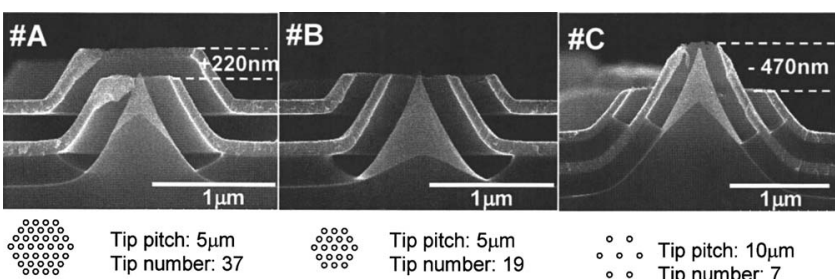

FIG. 1. Cross-sectional SEM images of the VDG-FEAs with different heights of the focusing electrode. Focusing electrode heights are $+220 \mathrm{~nm}$ (A), $0 \mathrm{~nm}(\mathrm{~B})$, and $-470 \mathrm{~nm}$ (C) relative to the extraction electrode. Tip arrangements are also shown.

out focusing operation. The focusing electrode voltage $\left(V_{f}\right)$ was the same as $V_{\text {ext }}$. Each threshold voltage was estimated about $20 \mathrm{~V}$ and $I_{a}$ achieved several microamperes at $V_{\text {ext }}$ of $60 \mathrm{~V}$. Excellent emission characteristics were obtained for each VDG-FEA.

\section{B. Focusing operation}

Figure 3(a) shows the dependence of the substrate current $\left(I_{\text {sub }}\right)$ on $V_{f}$ and Fig. 3(b) shows the dependence of $I_{a}$ on $V_{f}$. $V_{\text {ext }}$ was fixed to $60 \mathrm{~V}$ and $V_{f}$ was varied from 60 down to $0 \mathrm{~V}$. As shown in Fig. 3(a), $I_{\text {sub }}$ drastically decreased with decreasing $V_{f}$ for A and B. On the other hand, in C, $I_{\text {sub }}$ was almost independent of $V_{f}$. For example, at $V_{f}=0 \mathrm{~V}, I_{\text {sub }}$ was reduced to about $0.2 \%$ of the unfocused current $\left(V_{f}=60 \mathrm{~V}\right)$ for $\mathrm{A}$ and $3 \%$ of the unfocused current for B. In contrast to them, $I_{\text {sub }}(1.44 \mu \mathrm{A})$ was maintained over $30 \%$ of the unfocused current $(4.64 \mu \mathrm{A})$ at $V_{f}=0 \mathrm{~V}$ for $\mathrm{C}$. This result indicates that the electric field at the tip of $\mathrm{C}$ can be maintained still high even under focusing operation around $V_{f}=0 \mathrm{~V}$. It was considered that the substrate current reduction under the focusing operation for A and B was caused by the relaxation of the electrical field at the tip. As shown in Fig. 3(b), the dependences of $I_{a}$ on $V_{f}$ are similar to those of $I_{\text {sub }}$. However, each $I_{a}$ drastically decreased in the strong focusing region below $5 \mathrm{~V}$ for every sample. $I_{a}$ was reduced to less than $0.01 \%$ of the unfocused current even for C. In the strong

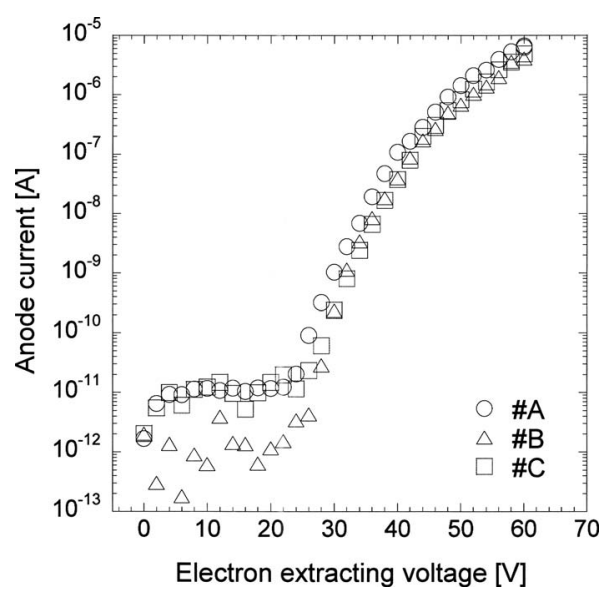

FIG. 2. Emission characteristics of the VDG-FEAs with different focusing electrode heights. The voltages of the focusing electrode and the extraction electrode were the same. (a)

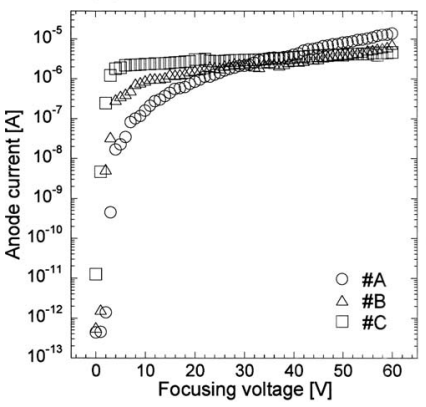

(b)

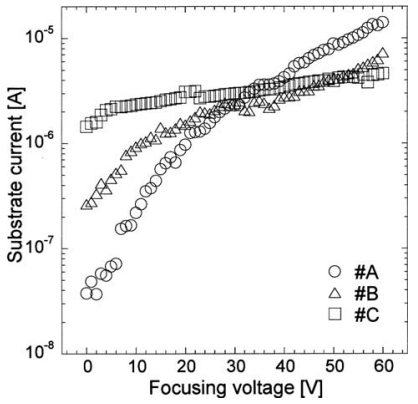

FIG. 3. Substrate current (a) and anode current (b) as a function of the focusing voltage. The extraction electrode was constantly biased at $60 \mathrm{~V}$.

focusing region, almost all electrons emitted from the each emitter were considered to return to the extraction electrode by the saddle point, which was formed by the low focusing potential. The detail will be discussed later.

Figure 4 shows the Fowler-Nordheim (FN) plots of the relationship between $I_{\text {sub }}$ and $V_{f}$ to estimate the influence of the focusing electrode voltage on the electric field at the tip. The negative slope parts appeared in the FN plots of the focusing characteristics for A and $\mathrm{B}$. These negative slope parts mean that the emission current emitted from the emitter (not the anode current, but the substrate current) depends on the focusing voltage in the VDG-FEA structure. On the other hand, $\mathrm{C}$ had no negative slop. This means that the influence of $V_{f}$ on the electric field at the tip is almost removed by the lowering focusing height for $\mathrm{C}$.

The potential profile of each sample was calculated by using the simulator (PARTICLE STUDIO, cst GmbH). Figure 5 shows the potential profiles along the direction from the emitter tip to the anode under the following strong focusing conditions; the substrate voltage $\left(V_{\text {sub }}\right), V_{\text {ext }}, V_{f}$, and the electric field from the anode to the tip are $0 \mathrm{~V}, 60 \mathrm{~V}, 5 \mathrm{~V}$, and $5000 \mathrm{~V} / \mathrm{cm}$, respectively. Position $0 \mu \mathrm{m}$ indicates the position of the emitter tip. The voltage of the extraction electrode rapidly increases the potential near the tip. The insert shows the potential profiles above the tip in $10 \mathrm{~nm}$ area. It is found that the electric field relaxation occurs in A and B due to the

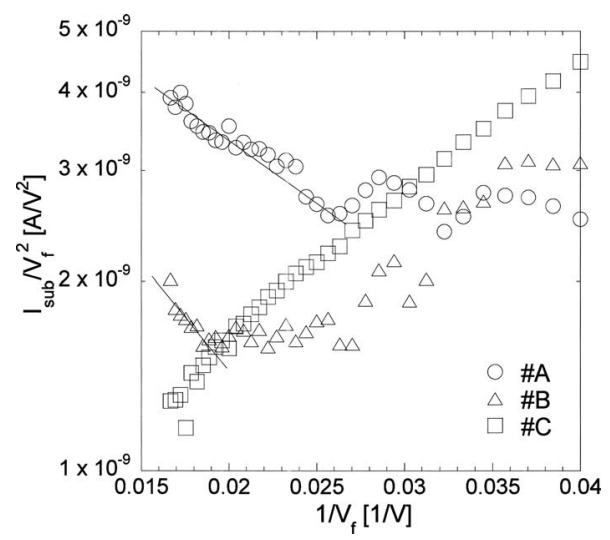

FIG. 4. Fowler-Nordheim plots between the substrate currents $\left(I_{\text {sub }}\right)$ and the focusing voltages $\left(V_{f}\right)$ for each sample. 


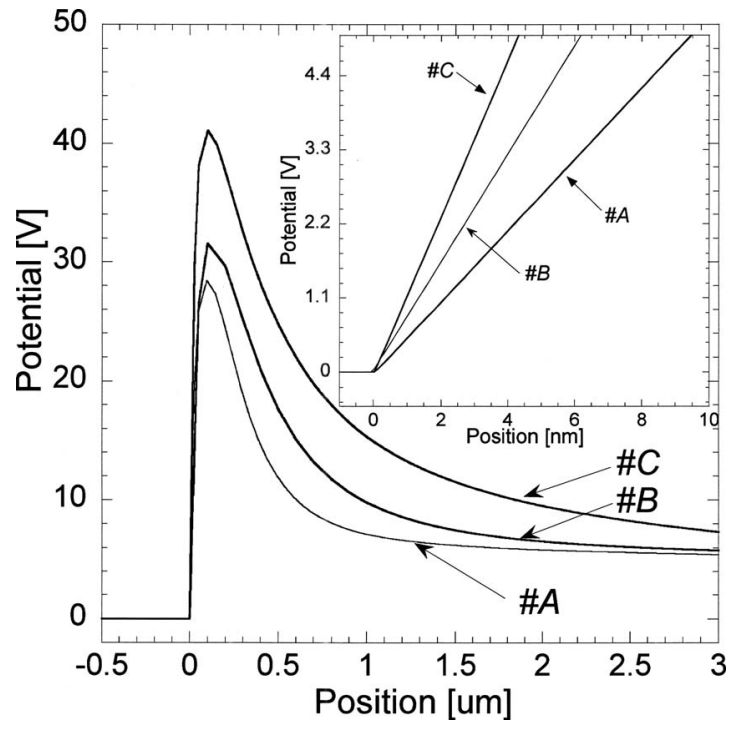

FIG. 5. Potential profiles along the direction from the emitter to the anode for each sample. The insert shows the magnified potential profiles in $10 \mathrm{~nm}$ area above the tip.

low focusing voltage. In contrary to it, it is found that the electric field for $\mathrm{C}$ is maintained, indicating that the structure of $\mathrm{C}$ is very effective for electron emission even under the focusing operation. These simulated results well agree with the experimental characteristics.

\section{Potential barrier}

As shown in Fig. 5, the potential above a few micrometers apart from the tip is suddenly decreased by the low voltage of the focusing electrode for every sample. These potential barriers make the electrons emitted from the emitter return back to the extraction electrode. Figures 6(a) and 6(b) show the ration $\left(I_{\text {ext }} / I_{\text {sub }}\right)$ of the extraction electrode current $\left(I_{\text {ext }}\right)$ to $I_{\text {sub }}$ and $I_{a} / I_{\text {sub }}$, respectively. Under the strong focusing operation below $5 \mathrm{~V}, I_{\text {ext }} / I_{\text {sub }}$ and $I_{a} / I_{\text {sub }}$ drastically increases and decreases, respectively, indicating that almost all electrons emitted from the emitter return back to the extraction electrode. As shown in Figs. 6(a) and 6(b), we can confirm that almost all electrons cannot reach the anode and return back to the extraction electrode at $V_{f}=0 \mathrm{~V}$ due to the poten-
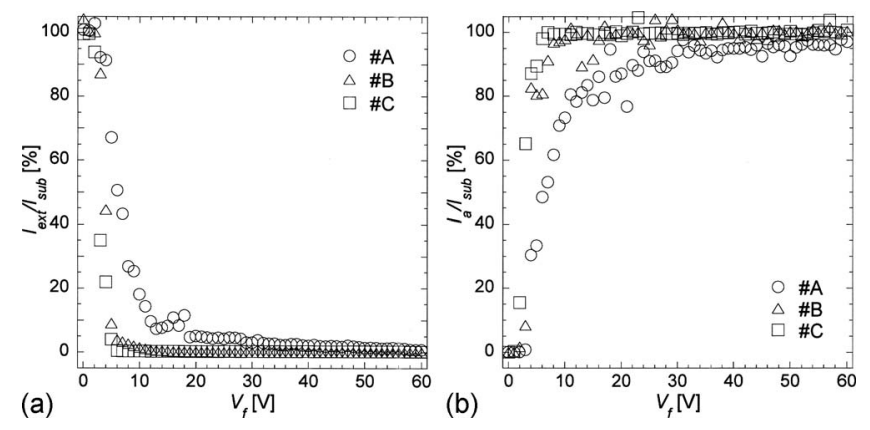

FIG. 6. Ratio $\left(I_{\text {ext }} / I_{\text {sub }}\right)$ of the extraction electrode current to the substrate current (a) and the ratio $\left(I_{a} / I_{\text {sub }}\right)$ of the anode current to the substrate current (b) as a function of the focusing voltage.

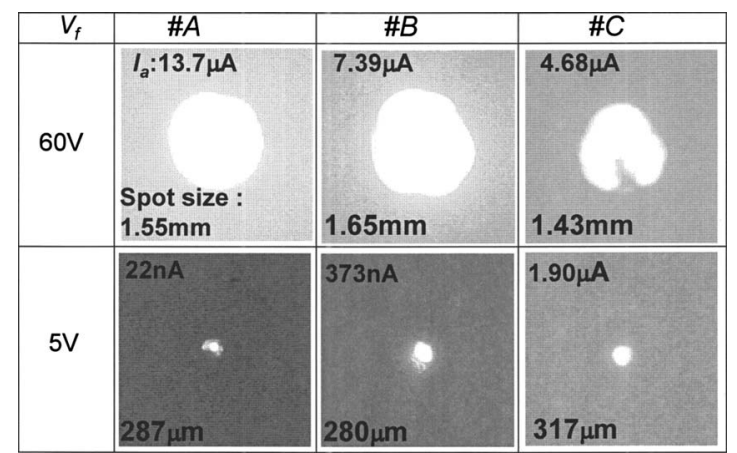

FIG. 7. Anode currents and beam spot sizes as measured on the anode screen at the focusing voltages of 60 and $5 \mathrm{~V}$.

tial barrier. Especially, the focusing electrode arrangement in A easily forms the potential barrier so that a number of electrons were returned back to the extraction electrode even under weak focusing operation. The results in Figs. 6(a) and 6(b) indicate that lowering the focusing electrode height like $\mathrm{B}$ and $\mathrm{C}$ is effective to reduce the influence of potential barrier. However, the formation of the potential barrier is not avoidable in double-gated FEA structures, because a focusing electrode essentially works as an electron mirror. In order to overcome such a retarding effect by the potential barrier, equipping with another focusing lens which does not form an electron mirror is necessary. For example, an acceleration type Einzel lens, accompanied by increasing the anode voltage, is an effective candidate.

\section{Focusing characteristics}

Figure 7 shows the beam spots displayed on the anode screen under focusing condition of $V_{f}=5 \mathrm{~V}$ and unfocused condition of $V_{f}=60 \mathrm{~V}$. The anode currents are also shown in Fig. 7. The anode and extraction voltages were fixed at $1 \mathrm{kV}$ and $60 \mathrm{~V}$, respectively. Each spot size was reduced to about $1 / 5$ of the unfocused one for each sample, indicating that every sample can produce a good focused electron beam. In the case of $\mathrm{C}$, the spot size was reduced from $1.43 \mathrm{~mm}$ to $317 \mu \mathrm{m}$ with reducing $V_{f}$ from 60 to $5 \mathrm{~V}$. The divergence angle of electron beam is estimated to be reduced from 216 to $50 \mathrm{mrad}$. Furthermore, the anode current was maintained at $1.9 \mu \mathrm{A}$ even under the focusing operation of $V_{f}=5 \mathrm{~V}$. This anode current is about 84 times and 5 times larger, compared with $\mathrm{A}$ and $\mathrm{B}$, respectively. Lowering the focus electrode height is quite effective for preventing the current to decrease for double-gated field emitters.

\section{CONCLUSIONS}

The dependence of the focusing characteristic on the focusing electrode height for VDG-FEAs was investigated. Three kinds of VDG-FEAs with different focusing electrode heights have been fabricated by using etch-back technique. Focusing effect was confirmed in every sample. However, for the samples with $h f=200 \mathrm{~nm}$ and $h f=0 \mathrm{~nm}$, the current 
to the screen was strongly reduced. On the other hand, the samples with $h f=-470 \mathrm{~nm}$ the anode current of about $1.9 \mu \mathrm{A}$ was maintained, even in applying $5 \mathrm{~V}$ to the focusing electrode, and the anode current was about 84 times and 5 times larger, compared with the samples with $h f=220 \mathrm{~nm}$ and $h f=0 \mathrm{~nm}$ under the same focusing condition. These results indicate that both the excellent endurance against the low potential of the focusing electrode and the superior focusing characteristic are realized for the optimized VDGFEA structure.

\section{ACKNOWLEDGMENTS}

The work was supported, in part, by Shizuoka University 21st COE (Center of Excellence) Program and Grant-in Aid for Scientific Research from the Ministry of Education, Culture, Sports, Science and Technology, Japan.

${ }^{1}$ R. M. Mobley and J. E. Boers, IEEE Trans. Electron Devices 38, 2383 (1991).

${ }^{2}$ J. Itoh, Y. Tohma, K. Morikawa, S. Kanemaru, and K. Shimizu, J. Vac. Sci. Technol. B 13, 1968 (1995).

${ }^{3}$ A. Hosono, S. Kawabuchi, S. Horibata, and S. Okuda, J. Vac. Sci. Technol. B 17, 575 (1999).

${ }^{4}$ T. Soda, M. Nagao, C. Yasumro, S. Kanemaru, T. Sakai, N. Saito, Y. Neo, T. Aoki, and H. Mimura, Jpn. J. Appl. Phys. 47, 5252 (2008).

${ }^{5}$ Y. Neo et al., Appl. Phys. Express 1, 053001 (2008).

${ }^{6}$ G. Hashiguchi, H. Mimura, and H. Fujita, Jpn. J. Appl. Phys., Part 2 35, L84 (1996).

${ }^{7}$ D. Nicolaescu, M. Nagao, T. Sato, V. Filip, S. Kanemaru, and J. Itoh, J. Vac. Sci. Technol. B 23, 707 (2005).

${ }^{8}$ T. Sato, S. Yamamoto, M. Nagao, T. Matsukawa, S. Kanemaru, and J. Itoh, J. Vac. Sci. Technol. B 24, 1589 (2003). 